\title{
Targeting MII1 H3K4 methyltransferase activity to guide cardiac lineage specific reprogramming of fibroblasts
}

\author{
Liu Liu ${ }^{1}$, Ienglam Lei, ${ }^{1,2}$, Hacer Karatas ${ }^{3,4,5}$, Yangbing Li ${ }^{3,4,5}$, Li Wang ${ }^{6,7,8}$, Leonid Gnatovskiy ${ }^{1}$, Yali Dou ${ }^{9}$, \\ Shaomeng Wang ${ }^{3,4,5}$, Li Qian ${ }^{6,7,8}$, Zhong Wang ${ }^{1}$ \\ ${ }^{1}$ Department of Cardiac Surgery, Frankel Cardiovascular Center, The University of Michigan, Ann Arbor, MI, USA; ${ }^{2}$ Faculty of \\ Health Sciences, University of Macau, Macau SAR, China; ${ }^{3}$ Department of Internal Medicine, University of Michigan School \\ of Medicine, Ann Arbor, MI, USA; ${ }^{4}$ Department of Pharmacology, University of Michigan School of Medicine, Ann Arbor, \\ MI, USA; ${ }^{5}$ Department of Medicinal Chemistry, University of Michigan College of Pharmacy, Ann Arbor, MI, USA; \\ ${ }^{6}$ Department of Pathology and Laboratory Medicine, University of North Carolina, Chapel Hill, NC, USA; ${ }^{7}$ McAllister Heart \\ Institute University of North Carolina, Chapel Hill, NC, USA; ${ }^{8}$ Lineberger Comprehensive Cancer Center, University of North \\ Carolina, Chapel Hill, NC, USA; ${ }^{9}$ Department of Pathology, University of Michigan Medical School, 1301 Catherine, \\ Ann Arbor, MI, USA
}

Generation of induced cardiomyocytes (iCMs) directly from fibroblasts offers a great opportunity for cardiac disease modeling and cardiac regeneration. A major challenge of $\mathrm{iCM}$ generation is the low conversion rate. To address this issue, we attempted to identify small molecules that could potentiate the reprogramming ability towards cardiac fate by removing inhibitory roadblocks. Using mouse embryonic fibroblasts as the starting cell source, we first screened 47 cardiac development related epigenetic and transcription factors, and identified an unexpected role of H3K4 methyltransferase MII1 and related factor Men1 in inhibiting iCM reprogramming. We then applied small molecules (MM408 and MI503) of MIl1 pathway inhibitors and observed an improved efficiency in converting embryonic fibroblasts and cardiac fibroblasts into functional cardiomyocyte-like cells. We further observed that these inhibitors directly suppressed the expression of MIl1 target gene Ebf1 involved in adipocyte differentiation. Consequently, MIl1 inhibition significantly decreased the formation of adipocytes during iCM induction. Therefore, MIl1 inhibitors likely increased iCM efficiency by suppressing alternative lineage gene expression. Our studies show that targeting MII1 dependent H3K4 methyltransferase activity provides specificity in the process of cardiac reprogramming. These findings shed new light on the molecular mechanisms underlying cardiac conversion of fibroblasts and provide novel targets and small molecules to improve iCM reprogramming for clinical applications.

Keywords: adipocyte; cardiomyocyte; Cardiac reprogramming; H3K4 methyltransferase; M111 inhibitor

Cell Discovery (2016) 2, 16036; doi:10.1038/celldisc.2016.36; published online 11 October 2016

\section{Introduction}

Heart disease is the leading cause of death in the United States and around the world [1]. On heart injuries, such as myocardial infarction, millions of cardiomyocytes undergo irreversible necrosis and infarct areas are replaced with fibroblasts and further differentiate into nonfunctional tissues like fibrotic

Correspondence: Zhong Wang

Tel: +1-734-763-3691

E-mail: zhongw@med.umich.edu.

Received 28 August 2016; accepted 5 September 2016 scar [2] or adipose tissue [3, 4]. Recently, targeting fibroblasts by introduction of three transcription factors Gata4, Mef2C and Tbx5 (G, M and T), and reprogramming them into cardiomyocyte-like cells have shown therapeutic potential [5-8]. However, direct reprogramming of fibroblasts into functional cardiomyocytes with high efficiency remains a challenge.

Numerous strategies have been applied to achieve better efficiency of induced cardiomyocyte (iCM) reprogramming. One strategy is to supply/introduce additional transcription factors to the cocktail. It has been shown that adding Hand2 [9], Nkx2-5 [10], 
Mesp1, MyoCD or Baf60c [11] can increase the reprogramming efficiency and/or improve functional properties of iCMs. Although multiple combinations have been used for reprogramming, Mef2C, Gata4 and Tbx5 (MGT) seem to be irreplaceable. Furthermore, stoichiometric expression of $\mathrm{G}, \mathrm{M}, \mathrm{T}$ proteins also affects the efficiency of MGT-mediated reprogramming [7]. Other strategies include application of Akt1 [12] or TGF-beta and Rho-associated kinase [13] to inhibit pro-fibrotic signaling. Two microRNAs, mir-1 and mir-133, when used in combination with transcription factors, could also increase iCM reprogramming efficiency [13-15].

Cardiac reprogramming is a process involving extensive epigenetic changes [16]. Therefore, modulating epigenetic changes is an effective strategy to improve efficiency. Successful examples include the application of inhibitors of Ezh2 methyltransferase and G9a histone methyltransferase [17] and shRNAmediated knockdown of Bmil, a regulatory component of polycomb complex involved in gene silencing [18].

Another potential novel strategy to achieve more specificity is to block conversion of fibroblasts into non-myocytes during iCMs induction. The induction of unexpected cell types in reprogramming appears to be rather common. It has been observed that Ascl1 promotes both neuron and myocyte identities in direct reprogramming from fibroblasts to neurons [19]. A recent study also shows that Yamanaka factor-directed iPSC reprogramming induces extraembryonic endoderm stem cells [20]. During iCM reprogramming, the binding of transcription factor to non-cardiac specific promoter can induce non-cardiac gene expression and likely leads to differentiation of unexpected cell types $[21,22]$. Therefore, identifying epigenetic factors and related small molecules to block lineage conversion into non-cardiomycoyte cell types may in turn promote iCMs formation.

In this report, we used a gain-of-function screen to identify regulatory factors involved in $\mathrm{iCM}$ reprogramming, which revealed an unexpected role of M111 H3K4 methyltransferase and related co-activator Men1 in inhibiting iCM formation. We demonstrated that addition of M111 inhibitor MM408 converted embryonic fibroblasts into functional cardiomyocytelike cells with a much higher efficiency. Furthermore, we identified Ebf1, a key factor involved in adipocyte differentiation, as a target of M111 during iCM reprogramming. Consequently, we observed that MM408 treatment during reprogramming inhibited adipocyte related gene expression and adipocyte formation and guided more cells into cardiomyocyte lineage. These findings provide novel insights into the molecular mechanisms of iCM reprogramming and identify small molecules for improved iCM generation.

\section{Results}

Gain-of-function screen identified menin1 (Men1) as a barrier to iCM reprogramming

To identify potential transcription and epigenetic regulators of iCM reprogramming, we employed a gain-of-function approach to explore the function of 47 genes (Figure 1a). On the basis of RNA-seq data of cardiac cell differentiation [23], we cloned 47 transcription and epigenetic regulators highly expressed in the cardiac progenitor and neonatal cardiomyocytes stage into a pCW57.1 based lentivirus vector pool (Supplementary Table S1). The individual genes were then transduced into mouse embryonic fibroblasts (MEFs) from a transgenic $\alpha$-muscle heavy chain ( $\alpha \mathrm{MHC}$ )-green fluorescent protein (GFP) reporter mouse $[5,7]$ together with retroviruses expressing polycistronic Mef2c/Gata4/Tbx5 (MGT) [7]. Ten days after transduction, activation of GFP was used to evaluate the efficiency of reprogramming (Figure 1b). For the MGT plus empty pCW57.1 group, we observed $11 \%$ GFP positive cells as reported previously, indicating similar successful rate for reprogramming (Figure 1c). Furthermore, we also observed the enhancing effect of Hand2, Mesp1 and Baf60c as reported $[9,11]$ (Figure 1b). Among the 47 candidate regulators, overexpression of epigenetic regulators Men1 and Suv39h1 resulted in fivefold and threefold reduction in $\alpha \mathrm{MHC}-\mathrm{GFP}+$ cells (Figure 1b). Flow cytometry detected 3.9 and $4.5 \%$ of $\alpha$ MHC-GFP+ cells in MGT+ Men1 and MGT+ Suv39h1 groups, respectively (Figure 1c). Quantitative real-time PCR (QPCR) results indicated that key cardiac genes Actcl and Tnnt2 were reduced following Men1 or Suv39h1 overexpression (Figure 1d). These results showed that Men1 and Suv39h1 were barriers to iCM reprogramming.

\section{Men1 inhibited reprogramming through Mll1}

Men1 is related to histone marks $\mathrm{H} 3 \mathrm{~K} 9 \mathrm{me} 3$ and $\mathrm{H} 3 \mathrm{~K} 4 \mathrm{me} 3$ [24, 25], and Suv39h1 is a regulator for H3K9me3 [26] (Figure 2a). As H3K9me3 has been known as a repressive marker, we postulated that Men1 might inhibit reprogramming through activating H3K9 methyltransferase activity. Therefore, we applied Suv39h1 inhibitor chaetocin [27] and Men1 inhibitor MI503 [28] in our reprogramming assays. 
Surprisingly, we did not observe any improvement in reprogramming with addition of Suv39h1 inhibitor chaetocin, but observed 1.5-fold increase with Men1 inhibitor MI503 (Figure 2b). Because MI503 targets Men1 by blocking the binding of Men1 to the M111 and M112 complex (Figure 2a) [28], Men1 likely affected reprogramming by activating M111/2-related H3K4 methyltransferase activity, instead of repressing H3K9me methyltransferase activity.

Histone H3K4 methylations are generally associated with gene activation, therefore our finding that inhibiting the activity of M1l1 H3K4 methyltransferase enhanced cardiac reprogramming was surprising. To confirm that MI503 increased reprogramming through inhibiting M111-related H3K4me3 activity, we applied a novel Mll1 complex-specific inhibitor in our system.
MM408 [29] compound is a modified version of the previously reported MM401 [30] that disrupts the interaction of WDR5 with Mll1 with higher binding affinity (Figure 2c). It specifically inhibits Mll1-related H3K4 methyltransferase activity without affecting the activities of other M1l family proteins. Indeed, MM408 inhibitor increased reprogramming efficiency in a dose dependent manner (Figure 2d). Overall, $17.5 \mu \mathrm{M}$ or $35 \mu \mathrm{M}$ MM408 resulted in the highest iCM conversion rate, whereas $70 \mu \mathrm{M}$ MM408 resulted in potential toxicity and lowered the conversion rate. Also, $17.5 \mu \mathrm{M}$ MM408 led to a significantly higher iCM conversion rate compared with optimized $1 \mu \mathrm{M}$ MI503 (Figure 2e). In addition, Mll1 shRNA knockdown also resulted in elevated iCM conversion rate (Figure 2e). Collectively, these results suggested that inhibiting M111 activities a

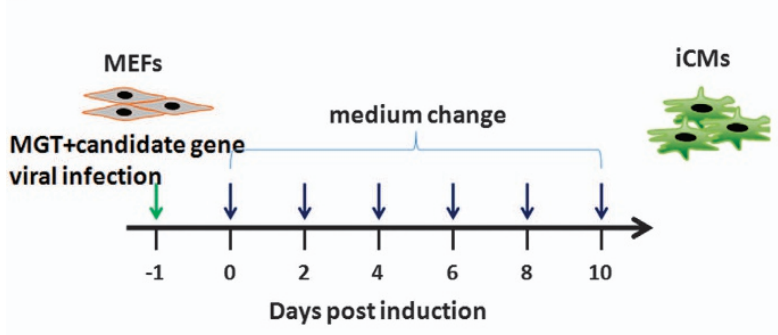

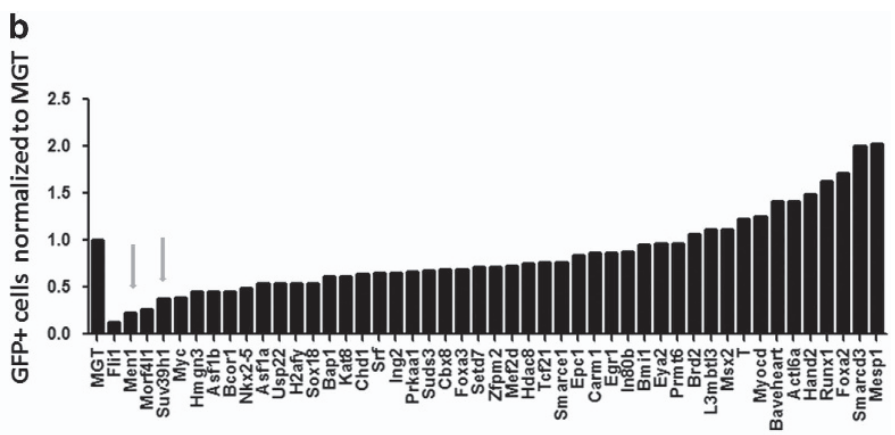

c

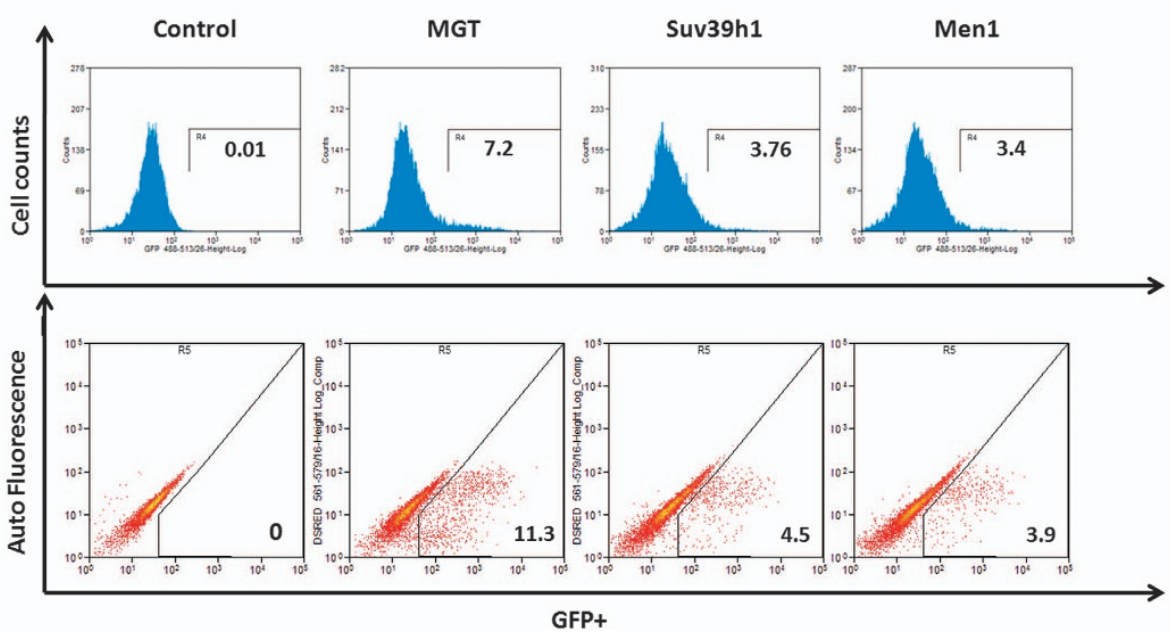

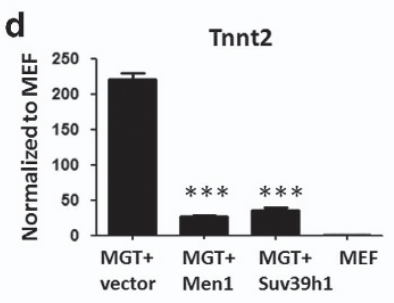

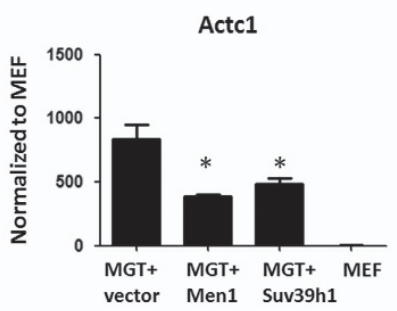

Figure 1 Gain-of-function screen identified Men1 as a barrier of iCM reprogramming. (a) A schematic diagram of the gain-of-function screen strategy. Cloned individual gene was transduced into MEFs derived from a transgenic $\alpha$ MHC-GFP reporter mouse together with retroviruses expressing polycistronic MGT. Medium was changed every 2 days. Activation of GFP was evaluated 10 days after transduction by flow cytometry. (b) Column graph of normalized percentages of $\alpha \mathrm{MHC}-\mathrm{GFP}+$, green arrow pointing at the fold change of Men1 and Suv39h1. (c) Representative flow cytometry results of Men1 and Suv39h1 groups shown by histogram (upper panel) and flow plots (lower panel). For the MGT plus empty pCW57.1 group, 11\% GFP positive cells were detected by flow cytometry. For MGT+ Men1 and MGT+ Suv39h1 groups, 3.9 and $4.5 \%$ of $\alpha \mathrm{MHC}-\mathrm{GFP}+$ cells were detected, respectively. (d) Relative expression of cardiomyocytes marker genes Tnnt2 and Actc1 in MEFs infected with MGT combined with Men1, Suv39h1 or empty vector after 10 days. Untransduced MEFs were used as a negative control (MEF). Error bars indicate mean \pm s.e.m.; ${ }^{*} P<0.05$, ${ }^{* *} P<0.01$, ${ }^{\star * \star} P<0.001$. 
by applying small molecules MI503 and MM408 increased the reprogramming efficiency.

\section{Mll1 inhibitors significantly enhanced cardiac reprogramming}

We next investigated the effect of Mll1 inhibition on the functional properties of iCMs. As MM408 resulted in the highest iCM conversion rate compared with other inhibitors, we focused on MM408 in our following experiments. Owing to its near highest iCM conversion rate and low cell toxicity, $17.5 \mu \mathrm{M}$ MM408 was used in these experiments (Figure 2). We first performed molecular characterizations of the MM408-treated and untreated cells by examining the expression of a panel of sarcomeric, contractility and ion channel genes. The results revealed a higher expression of these functionally important cardiac genes especially Actc1, Scn5a and Myh6 in the MM408-treated group (Figure 3a). In addition, MM408-treated cells showed reduced expression of genes associated with the fibroblast lineage. To test whether treatment of MM408 promotes iCM maturation, we then examined the structural properties using immunostaining against cardiac Troponin $\mathrm{T}$ (cTnT) and sarcomeric $\alpha$-actinin, two specific components of sarcomere in cardiomyocyte. Immunostaining against these two proteins could clearly show the sarcomeric structure. Treatment with MM408 for 2 weeks significantly increased the number of cells with assembled sarcomeric structures compared a

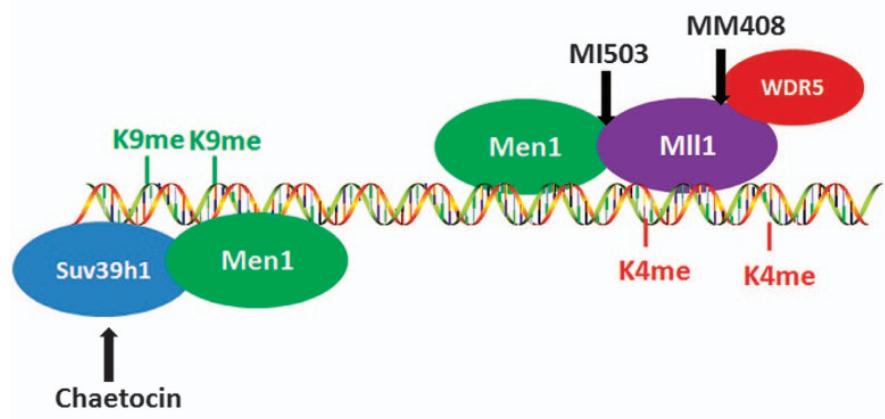

C<smiles>CC[C@H](NC(=O)[C@H](CCCNC(=N)NC)NC(=O)[C@](C)(CCCCNC(=O)[C@H](NC(=O)C(C)C)c1ccccc1)NC(=O)C(C)C)C(=O)NC</smiles>

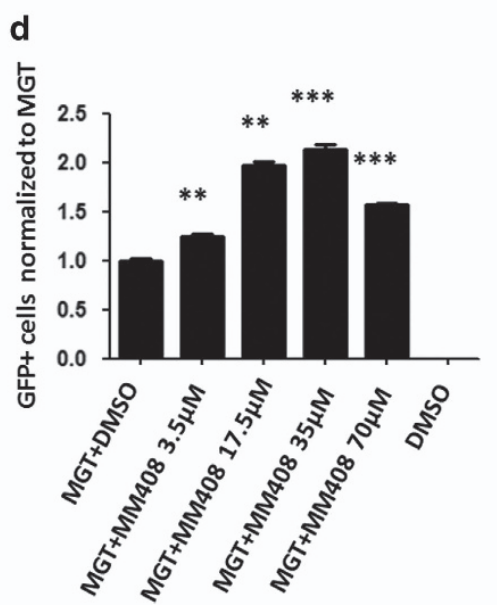

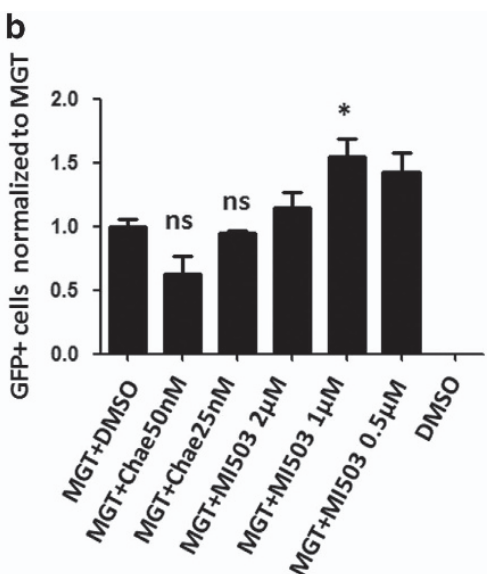

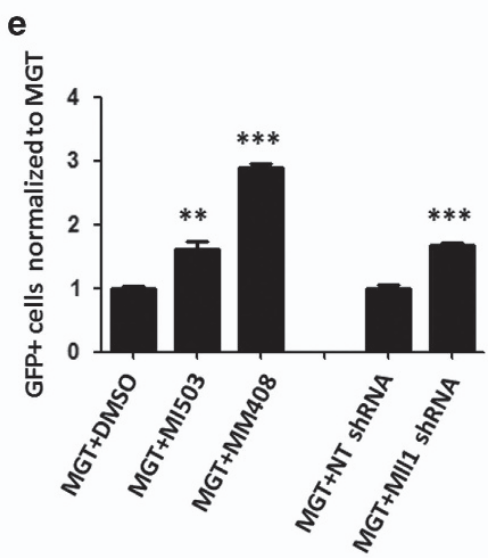

Figure 2 MII1 inhibitors significantly enhanced cardiac reprogramming. (a) A diagram depicting the interaction of Men1 with MII1 complex and Men1 with Suv39h1. The target sites of small molecules have been marked with arrows. (b) The effect of Suv39h1 inhibitor chaetocin and Men1 inhibitor MI503 treatment on iCM reprogramming. Relative fold changes of $\alpha M H C-G F P+$ were normalized to MGT induction with DMSO treatment. (c) The chemical structure of MM408. (d) Optimization of MII1 inhibitor MM408 treatment for improving reprogramming indicated by the fold change of $\alpha \mathrm{MHC}-\mathrm{GFP}+$ cells. (e) The effect of MI503, MM408 and MII1 shRNA on iCM reprogramming indicated by the fold change of $\alpha$ MHC-GFP+ cells in MGT-transduced MEFs. Non-targeting (NT) shRNA was used as control for Mll1 shRNA. Error bars indicate mean \pm s.e.m.; ${ }^{\star} P<0.05$, ${ }^{\star \star} P<0.01$, ${ }^{* * *} P<0.001$. 
a

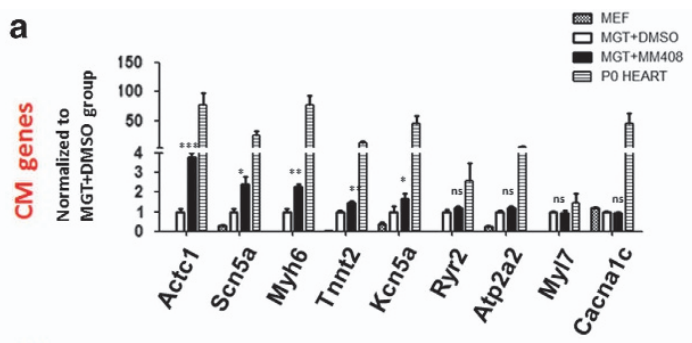

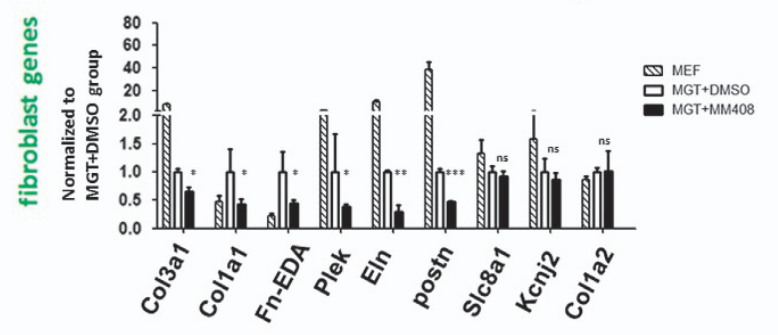

b

Sarcomeric Alpha

Actinin/DAPI merge magnification

MGT+DMSO

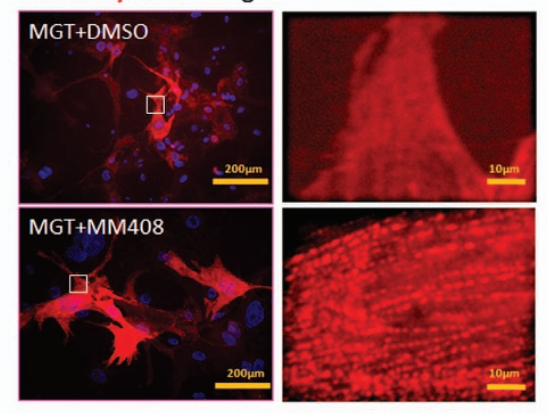

C
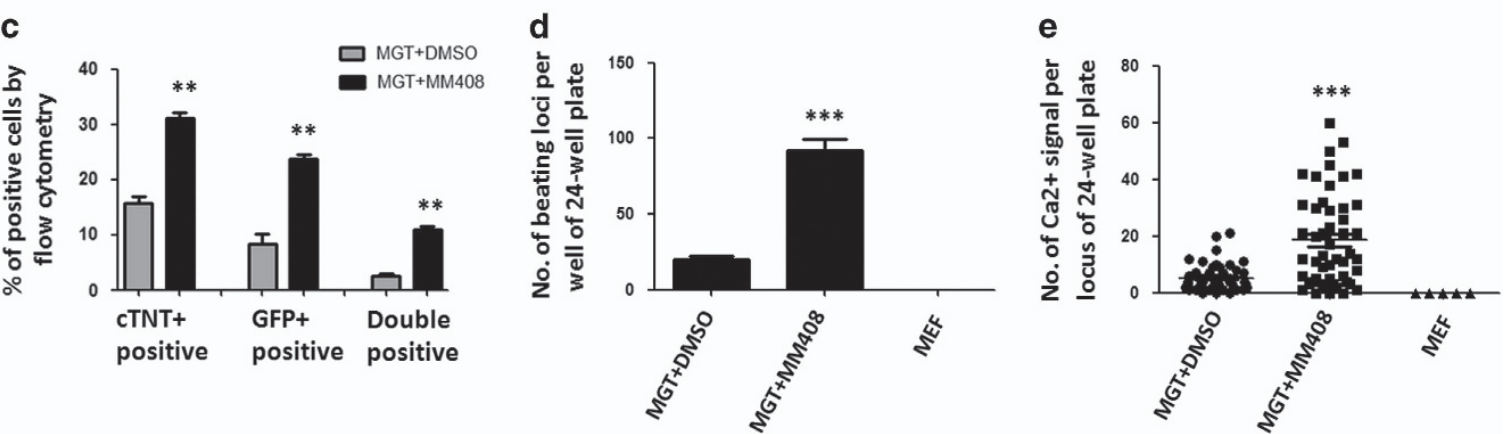

Figure 3 MII1 inhibitor MM408 improved functional properties of induced cardiomyocytes. (a) Relative expression of CM and fibroblast marker genes in neonatal whole heart isolated from postnatal day 0 (P0) mice and DMSO or MM408-treated iCMs 10 days after MGT transduction. Statistic analyses were performed between MM408- treatment and DMSO-treatment groups. (b) Immunocytochemistry (ICC) of cardiac markers $\alpha$-actinin of MGT-transduced cells with or without MM408 treatment by fluorescence microscopy $(400 \times)$. The right panels were enlarged areas from the left panels. (c) Quantification for $\alpha M H C-G F P+$ and cTnT+ cells or double positive cell detected by flow cytometry 10 days after transduction. (d) Quantification of the number of beating iCMs loci with indicated viral infection and small molecule treatment for 4 weeks $(n=10)$. (e) Quantification of spontaneous $\mathrm{Ca}^{2+}$ oscillations cells per field with indicated viral infection and small molecule treatment for 4 weeks $(n=50$ from 10 wells). Untransduced MEFs were used as a negative control (MEF). Error bars indicate mean \pm s.e.m.; ${ }^{*} P<0.05$, ${ }^{* *} P<0.01$, ${ }^{* * *} P<0.001$.

with the control group (Figure 3b; Supplementary Figure S1). Flow cytometric analyses further confirmed that MM408 induced higher cTnT expression. In particular, we observed that $25 \%$ of the cells became GFP positive and $30 \%$ of the cells became cTnT positive in MM408-treated group, more than twofold increase over non-MM408-treated group (Figure 3c). Notably, 10\% GFP and cTnT double positive cells were observed, indicating a more than threefold increase over controls.

Furthermore, we evaluated the presence of beating iCMs in our reprogramming assays using the beating protocol as reported [31]. Spontaneously contracting cells were apparent 3 weeks after MM408 treatment indicating that MM408 treatment significantly increased beating iCMs (Supplementary Movies S1 and S2). Approximately 92 beating loci per well were identified in MM408-treated group 4 weeks after transduction (Figure 3d). In contrast, only 20 beating loci were observed in non-MM408-treated group. To further assess the functionality of MM408-treated iCMs, we next analyzed intracellular $\mathrm{Ca}^{2+}$ flux by Rhod3 dye labeling 4 weeks after reprogramming. MM408-treated iCMs showed significantly higher spontaneous $\mathrm{Ca}^{2+}$ oscillations with various frequencies compared with control group (Figure 3e; Supplementary Movies S3 and S4), as previously reported in our reprogrammed iCMs $[7,18]$. By using neonatal cardiac fibroblast instead of MEFs transduced with MGT, we also observed more beating loci in MM408-treated 
a

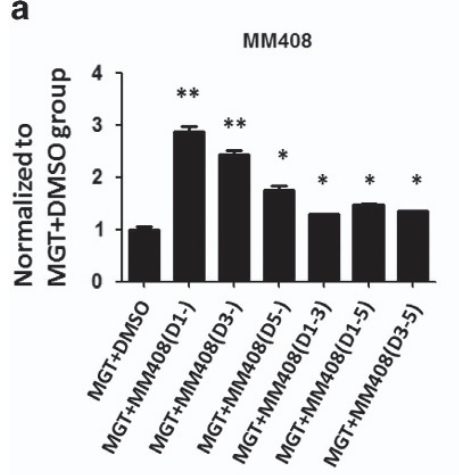

C

\begin{tabular}{|c|c|c|c|}
\hline Category & Term & Count & P Value \\
\hline $\begin{array}{l}\text { KEGG_PAT } \\
\text { HWAY }\end{array}$ & $\begin{array}{l}\text { mmu00480:Glutathione } \\
\text { metabolism }\end{array}$ & 18 & $2.75 E-05$ \\
\hline $\begin{array}{l}\text { KEGG_PAT } \\
\text { HWAY }\end{array}$ & $\begin{array}{l}\text { mmu04630:Jak-STAT signaling } \\
\text { pathway }\end{array}$ & 33 & $2.62 \mathrm{E}-04$ \\
\hline $\begin{array}{l}\text { KEGG_PAT } \\
\text { HWAY }\end{array}$ & $\begin{array}{l}\text { mmu00980:Metabolism of } \\
\text { xenobiotics by cytochrome P450 }\end{array}$ & 18 & 7.01E-04 \\
\hline $\begin{array}{l}\text { KEGG_PAT } \\
\text { HWAY }\end{array}$ & mmu05200:Pathways in cancer & 56 & $7.82 \mathrm{E}-04$ \\
\hline $\begin{array}{l}\text { KEGG_PAT } \\
\text { HWAY }\end{array}$ & $\begin{array}{l}\text { mmu03320:PPAR signaling } \\
\text { pathway }\end{array}$ & 20 & $8.71 E-04$ \\
\hline $\begin{array}{l}\text { KEGG_PAT } \\
\text { HWAY }\end{array}$ & $\begin{array}{l}\text { mmu04610: Complement and } \\
\text { coagulation cascades }\end{array}$ & 18 & 30.003160 \\
\hline $\begin{array}{l}\text { KEGG_PAT } \\
\text { HWAY }\end{array}$ & $\begin{array}{l}\text { mmu04920:Adipocytokine } \\
\text { signaling pathway }\end{array}$ & 16 & 0.006106 \\
\hline $\begin{array}{l}\text { KEGG_PAT } \\
\text { HWAY }\end{array}$ & mmu00982:Drug metabolism & 17 & 0.007676 \\
\hline $\begin{array}{l}\text { KEGG_PAT } \\
\text { HWAY }\end{array}$ & $\begin{array}{l}\text { mmu04060:Cytokine-cytokine } \\
\text { receptor interaction }\end{array}$ & & 0.007716 \\
\hline
\end{tabular}

b

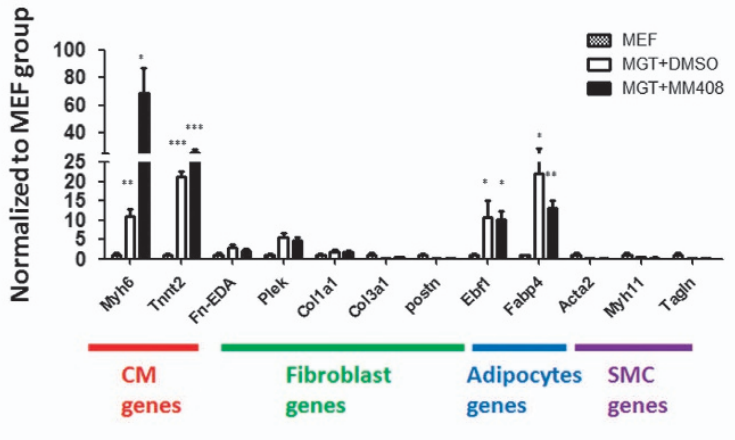

d

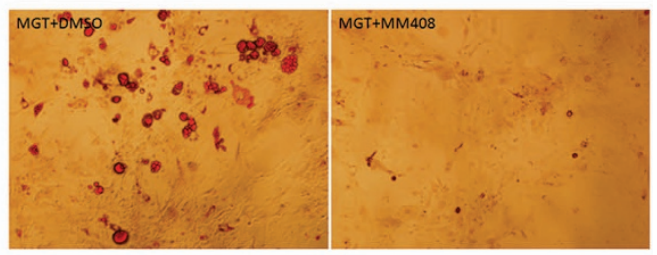

e.
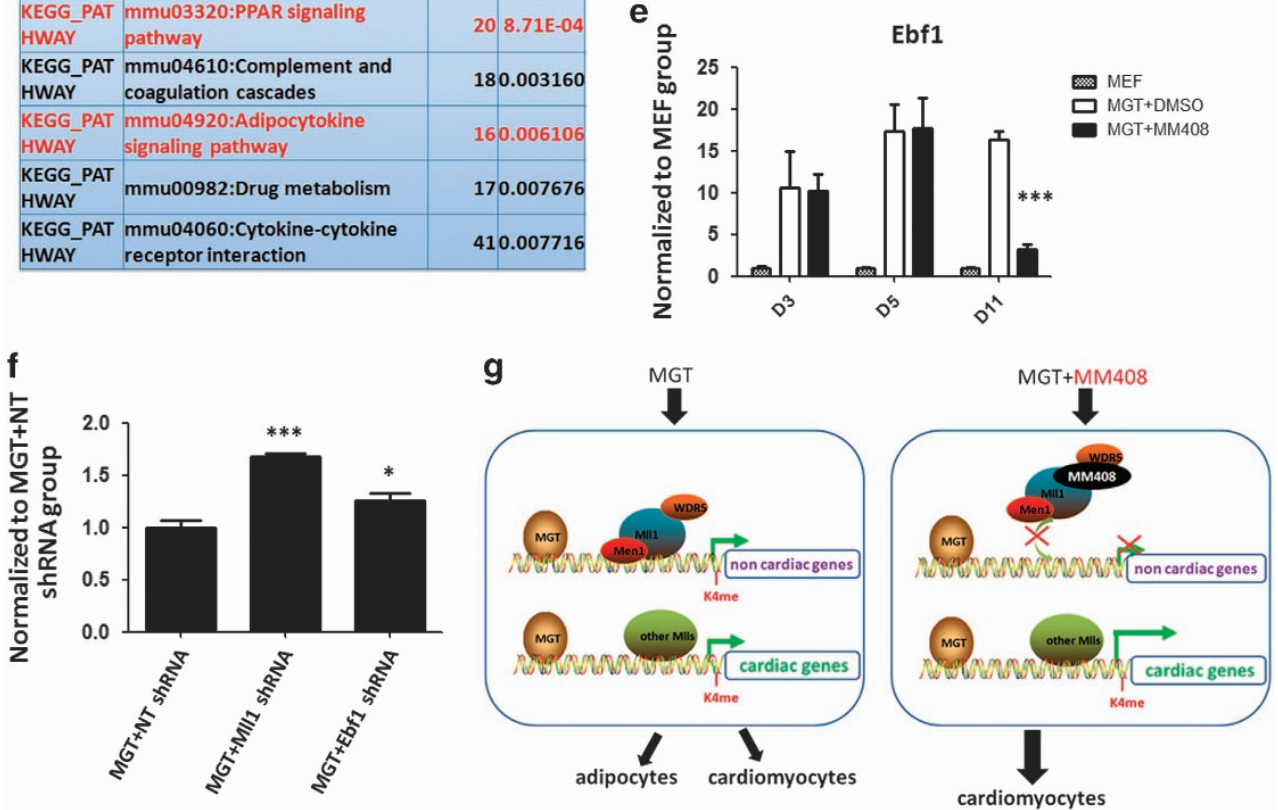

Figure 4 MII1 inhibitor guided cardiac reprogramming by suppressing ectopic adipocyte related gene expression. (a) The effect of timing and duration of WDR5 inhibitor MM408 treatment on iCM reprogramming, as shown by the fold change of $\alpha$ MHC-GFP+ cells after MGT transduction. MII1 inhibitor was added with various time durations after viral transduction: D1-, from day 1 until detection; D3-, from day 3 until detection; D5-, from day 5 until detection; D1-3, from day 1 to day 3; D1-5, from day 1 to day 5; D3-5, from day 3 to day 5. (b) Relative expression of cardiomyocyte (CM), fibroblast, adipocyte and smooth muscle cells (SMC) marker genes in early stage of iCM reprogramming with either MM408 or DMSO treatment 3 days after MGT transduction. Statistic analyses were performed between MEF with MM408-treated or DMSO-treated groups. Untransduced MEFs were used as a negative control (MEF). (c) Gene Ontology (GO) enrichment analysis of down-regulated genes in MII1 ${ }^{-1-}$ MEFs. The list shows enriched pathway ordered by $P$-value. The pathways related with Pparg and adipocyte lineage were marked in red. (d) Oil Red $O$ stain of adipocyte cells in MGT-transduced MEFs 14 days after transduction with or without MM408 treatment. (e) Expression of Ebf1 during reprogramming in MGT-transduced MEFs, with or without MM408 treatment. The $P$-value reflecting the statistical significant difference of the expression of Ebf1 at D11 was 0.0004 . Untransduced MEFs were used as a negative control (MEF). (f) The effect of Mll1 or Ebf1 knockdown on improving iCM efficiency indicated by the fold change of $\alpha M H C-G F P+$ cells. Non-targeting (NT) shRNA was used as a control for Mll1 or Ebf1 shRNA. (g) A working model of MM408 mediated cardiac reprogramming. Mll1 inhibitor MM408 promoted iCM conversion by suppressing adipocyte lineage gene expression. Error bars indicate mean \pm s.e.m.; ${ }^{*} P<0.05,{ }^{* *} P<0.01,{ }^{* * *} P<0.001$. 
group compared with non-MM408-treated group (Supplementary Figure S2; Supplementary Movies S5 and S6). These results revealed that MM408 not only increased cardiac gene expression, but also enhanced iCM function.

\section{Mll1 inhibitor guided cardiac reprogramming by suppressing adipocyte formation}

To characterize the cellular and molecular mechanisms of MM408/M111 action during iCM induction, we set out to determine the timing of Mll1 inhibitor-mediated effects on iCM reprogramming (Figure 4a). Fibroblasts were transduced with MGT on day 0, and MM408 compound was added to the culture medium at various time points. The most effective time-window was to add MM408 from day 1, resulting in three times as many GFP+ cells as negative control. These results indicated that MM408 exerted its effect from the initiation of reprogramming, consistent with the idea that epigenetic repatterning occurs at early stage of reprogramming [16, 18]. Therefore, we attempted to identify the direct target(s) of M111 in fibroblasts that mediate(s) its effect on iCM reprogramming.

Given the fact that $\mathrm{M}, \mathrm{G}$ and $\mathrm{T}$ are all potent transcription factors that regulate multiple biological processes during early embryonic development, it is likely that during MGT-mediated iCM formation, reprogramming factors not only activate cardiac specific gene expression but also induce non-cardiac lineage gene expression. To test this hypothesis, we examined the expression of fibroblast-, adipocyte- and SMC-related genes at the early stage of reprogramming (3 days post transduction). We found that in the early stage of reprogramming, overexpression of MGT in fibroblasts led to not only elevated cardiac gene expression but also increased adipocyte gene expression 3 days post transduction. In contrast, fibroblast or SMC-related genes in MM408-treated and DMSO-treated groups did not change (Figure 4b). By comparing the expression profiles of $\mathrm{M}^{111^{-1-}} \mathrm{MEFs}$ versus $\mathrm{M}_{111^{+/+}}$MEFs [32] with gene ontology analysis, we discovered that the down-regulated genes were highly related to adipogenesis and peroxisome proliferator-activated receptor pathways (Figure 4c). Consistent with this observation, in $\mathrm{M}_{111^{-1-}}$ MEFs, numerous genes related to those functions were down-regulated. More importantly, we observed adipocyte-like cells and increased adipocyte formation detected by Oil Red O stain 14 days after MGT transduction (Figure 4d). These results suggested that during reprogramming, ectopic expression of adipocyte lineage genes might interfere with the acquisition of cardiomyocyte cell fate in fibroblasts.

Therefore, we set out to test whether Mll1 inhibitors enhanced cardiac reprogramming by inhibiting adipocyte formation. On the basis of the fold change of expression and our previous report that M111 directly binds to Ebf1 gene locus in MEFs and embryonic stem cells [32, 33], we propose that adipogenesis-related Ebf1 could be a key target of M111 inhibitor in iCM reprogramming as Ebf1 expression was elevated during reprogramming (Figure 4e). In contrast, when MM408 was applied during iCM induction, expression of Ebf1 was dramatically decreased at day 11 post transduction (Figure 4e). Furthermore, adipogenesis marker genes Fabp4 and Pparg also exhibited a decreased expression after MM408 treatment (Supplementary Figure S3). Consistent with the decrease of adipogenesis-related gene expression, Oil Red $\mathrm{O}$ staining detected a significant decrease in adipocyte formation with MM408 treatment (Figure 4d). To directly test if blocking adipocyte lineage gene expression could promote iCM formation, we performed RNAi-mediated knockdown of Ebf1 and Mll1 during iCM formation. Indeed, knockdown of Ebf1 or Mll1 increased iCM reprogramming efficiency (Figure 4f), indicating that M111 inhibitor repressed adipocyte formation mainly through blocking the expression of Ebfl. Altogether, these results strongly suggested that inhibition of M111 promotes iCM conversion by suppressing adipocyte lineage gene expression (Figure 4g).

\section{Discussion}

We report here an unexpected role of M111 and related factor Men1 in cardiac reprogramming. Application of novel small molecules MM408 and MI503, inhibitors of M111 and Men1, resulted in much higher efficiency of iCM formation from embryonic fibroblasts. Mechanistically, these inhibitors directly suppressed the expression of Mll1 target gene Ebf1, a key gene involved in adipocyte differentiation. In parallel with Mll1 inhibition, we observed decreased formation of adipocytes in our iCM assays. Therefore, Mll1 inhibitors increased iCM efficiency likely through suppressing the expression of adipocyte lineage genes. Our studies show that targeting Mll1-related H3K4 methyltransferase activity with M111 inhibitors provides more specificity in the process of $\mathrm{iCM}$ induction.

Given the well-established antagonistic roles of TrxG and PcG complexes in development [34], our discoveries that inhibiting the activities of both $\mathrm{PcG}$ component Bmil and TrxG component Mll1 enhance 
iCM formation are surprising. However, our further studies indicate that M111 and Bmil exert their respective activating and repressive functions via distinct mechanisms [18]. Specifically, reduced Bmi1 expression leads to increased levels of the active histone mark $\mathrm{H} 3 \mathrm{~K} 4 \mathrm{me} 3$ and reduced levels of repressive H2AK199ub at cardiogenic loci, and de-repression of cardiogenic gene expression [18]. In contrast, as reported here, inhibiting Mll1 activity leads to decreased expression of genes involved in adipocyte formation and consequent low induction of adipocytes. Therefore, seemingly opposite functions of epigenetic factors may work together to facilitate reprogramming or transdifferentiation. Future studies will determine whether combined inhibition of Mll1 and Bmil could synergistically increase $\mathrm{ICM}$ conversion in a time-window dependent fashion.

To our knowledge, this is the first study to show that epigenetic factor/inhibitor can enhance cardiac reprogramming by blocking conversion of fibroblasts into non-myocyte cell types during iCMs induction. MM408, an inhibitor of M111-WDR5 interaction, is able to increase iCM formation by inhibiting adipogenesis. Our studies reported here point to a new direction in enhancing $\mathrm{iCM}$ conversion. Instead of boosting $\mathrm{iCM}$ conversion directly by increasing the cardiac gene expression, actively incorporating strategies to inhibit conversion of fibroblasts into other related cell types may also increase iCM formation.

Mechanistically, this MM408 mediated M111 inhibition is achieved, at least partially, by blocking the expression of M1l1 target Ebf1, a key gene involved in adipocytes differentiation [35]. Our discovery is consistent with a number of Mll1 studies in cell lineage development, which show that Men1 directly targets Pparg [36] through Mll to facility adipogenesis. Our results also show that in addition to Pparg, Ebf1 is also involved in adipocyte differentiation as a direct M111 target. Future research focusing on the epigenetic changes during the whole reprogramming process will reveal the mechanistic details of how Mll1 regulates cardiac reprogramming.

Our study shows that MGT not only induces iCM but also increases adipocyte formation. The molecular mechanism of MGT-mediated adipogenesis will be explored in the future. One potential reason might be that iCM and adipocytes are developmentally related. It has been shown that Myf5 positive myogenic precursors can differentiate into both adipocytes and muscle cells. Mature adipocytes [37] and adipocyte derived stem cells [38] possess the ability to differentiate into functional cardiomyocytes. These studies suggest close functional and original connections between adipocyte and muscle cell. Indeed, the observation that induction of unexpected cell types in reprogramming appears to be a common theme, as has been reported recently in neuron [19] and iPSC [20] reprogramming.

We expect that applications of chemical approaches for reprogramming and transdifferentiation represent a highly desirable strategy in heart regeneration to treat heart attack and associated heart failure. In fact, a couple of recent studies show the feasibility that cardiomyocyte-like cells can be obtained from embryonic fibroblasts with only small molecule treatment in vitro $[39,40]$. Future studies combining MM408 described here with small molecules and candidate molecules identified from other studies should convert fibroblasts into functional and more mature beating cardiomyocytes with high efficiency.

\section{Materials and Methods}

\section{Mouse lines}

The transgenic mice harboring GFP under control of $\alpha$-MHC promoter was used to derive MEFs as previous reported [7]. Another transgenic mice harboring mCherry under control of modified $\alpha$-MHC promoter was purchased from Jackson's lab (021577).

All animal-related procedures were approved by the Institutional Animal Care and Use Committee of the University of Michigan and are consistent with the National Institutes of Health Guide for Use and Care of Animals.

\section{Plasmids}

The retroviral polycistronic constructs vectors encoding mouse Mef2c, Gata4 and Tbx 5 in pMXs based vectors were constructed as described previously [7]. DNA fragment containing Mef2c, Gata 4 and Tbx 5 sequentially were separated by oligonucleotides encoding P2A and T2A peptides constructed into a polycistronic constructs vector. The shRNA lentivirus vector for Ebf1 (pGipZ, V2LMM-20601), M111 (pGipZ, V2LMM-96428) were from Vector Core of University of Michigan.

\section{Primary cell isolation}

MEFs (isolated at E13.5) were prepared as previously described. Briefly, embryos were harvested from transgenic mice of $\alpha$-MHC-GFP or $\alpha$-MHC-mCherry at 13.5 days post coitum followed by decapitation and removal of internal organs, including the heart. The tissue was minced and digested with TrypLE Express Enzyme (Thermo Fisher Scientific, Waltham, MA, USA). Cells were resuspended in MEFs medium ( $10 \% \mathrm{FBS}$ and $2 \mathrm{mM}$ L-glutamine contained DMEM medium) and plated onto one $10 \mathrm{~cm}$ dish per embryo. Cells were regularly passaged at 1:3 (passage 1). Passage 3 MEFs were used for reprogramming. 


\section{Inhibitor information}

Men1 inhibitor was from Active Biochem (MI503, A-1399), Suv39h1 inhibitor (chaetocin, sc-200893) was from Santa Cruz, M111/WDR5 inhibitor (MM408) was from Dr. Shaomeng Wang's lab.

\section{Retrovirus and lentivirus preparation}

Retrovirus vectors were packaged into Plat-E cells using Lipofectamine 2000 (Invitrogen, Carlsbad, CA, USA) to deliver $10 \mu \mathrm{g}$ in $1.5 \mathrm{ml}$ Opti-MEM (Invitrogen) to $80 \%$ confluent $10 \mathrm{~cm}$ plates of Plat-E cells with $6 \mathrm{ml}$ of Opti-MEM. 4-6 h later, Opti-MEM was changed into $10 \mathrm{ml}$ fresh MEFs medium. Forty-eight hours and seventy-two hours after transfection, viral medium was harvested twice and filtered through a $0.45-\mathrm{mm}$ cellulose filter. The harvested virus containing medium was added 1/5 vol of $40 \%$ PEG8000 solution to make a final concentration of $8 \%$ PEG8000. The mixture was kept at $4{ }^{\circ} \mathrm{C}$ overnight and spined at $3000 \mathrm{~g}, 4^{\circ} \mathrm{C}, 30 \mathrm{~min}$ to get concentrated white pellet. Fresh MEFs medium was added to resuspend virus. The viral supernatant was mixed with polybrene (Sigma, St Louis, MO, USA) to a final concentration of $8 \mu \mathrm{g} \mathrm{ml}^{-1}$.

Lentiviral vectors were packaged into HEK293T cells (ATCC) using Lipofectamine 2000 (Invitrogen) to deliver $10 \mu \mathrm{g}$ of the lentiviral backbone plasmid, $6 \mu \mathrm{g} \operatorname{psPAX} 2$, and $4 \mu \mathrm{g}$ pMD2.G in $1.5 \mathrm{ml}$ Opti-MEM (Invitrogen) to $80 \%$ confluent $10 \mathrm{~cm}$ plates of 293 cells with $6 \mathrm{ml}$ of Opti-MEM. 4-6 h later, Opti-MEM was changed into $10 \mathrm{ml}$ fresh MEFs medium. The virus was harvested as descried before.

\section{Direct conversion of fibroblasts to $i \mathrm{CMs}$}

Direct conversion of MEFs was completed using a protocol similar to that previously described [7]. Fresh fibroblasts were plated on tissue culture dishes at a density of 10000 cells $\mathrm{cm}^{-2}$ before transduction. Fibroblasts were infected with freshly made viral mixture containing polybrene (Sigma) $24 \mathrm{~h}$ post plating. Twenty-four hours later, the viral medium was replaced with induction medium composed of DMEM/199 (4:1) (Gibco, Waltham, MA, USA), 10\% FBS (Gemini, West Sacramento, CA, USA) and antibiotics (Gibco). Medium was changed every 2-3 days until cells were examined.

\section{Immunocytochemistry}

Samples were fixed using 4\% PFA with $0.1 \%$ TritonX-100. The following primary antibodies were used: mouse anti-cardiac Troponin T (Thermo Scientific, Waltham, MA, USA), mouse anti sarcomeric a-actinin (Abcam, Waltham, MA, USA). For Oil Red O staining, dishes were washed in PBS three times and cells fixed in $3.7 \%$ formaldehyde for $1 \mathrm{~h}$, followed by staining with Oil Red 0 for $1 \mathrm{~h}$; after staining, plates were washed twice in water and photographed. The final Oil Red $\mathrm{O}$ solution used consists of $60 \%$ original Oil Red O solution $(0.3 \%$ Oil Red O in $60 \%$ isopropanol (Sigma)) diluted with $40 \%$ DI water.

\section{Flow cytometry}

For fluorescence measurements only, data from 10000 single cell events were collected using a standard MoFlo Astrios flow cytometer (Immunocytometry Systems; Becton Dickinson,
Detroit, MI, USA). Data were analyzed using Summit (Becton Dickinson).

\section{Quantitative real time $P C R$ ( $Q P C R)$}

Total RNA was extracted using Trizol Reagent (Invitrogen) according to the manufacturer's instructions. RNA integrity was determined using formaldehyde denaturalization agarose gel electrophoresis. RNA concentrations were measured with the smartspec plus spectrophotometer (BioRad, Hercules, CA, USA). RNA was reverse transcribed using iScript cDNA Synthesis Kit (BioRad). QPCR was performed using StepOne Real-Time PCR System (Thermo Fisher Scientific).

Primer oligonucleotides were synthesized by Invitrogen and are listed in Supplementary Table S2.

\section{Statistical analysis}

Results were presented as mean \pm s.e.m. Statistical significance between groups was analyzed by one-way ANOVA followed by the Student-Newman-Keuls multiple comparisons tests. A $P$-value of $<0.05$ was considered significant. Each experiment was performed at least twice.

\section{Conflict of Interest}

The authors declare no conflict of interest.

\section{Acknowledgements}

This work was supported by the National Institutes of Health Grant HL109054, an Inaugural Award from the Samuel and Jean Frankel Cardiovascular Center, University of Michigan, a Pilot Award from the Joint Institute of University of Michigan Health System and Peking University Health Science Center (to WZ), AHA Scientist Development Grant 13SDG17060010, the Ellison Medical Foundation (EMF) New Scholar Grant AG-NS-1064-13 (to LQ) and the National Institutes of Health Grant R01CA177307 (to SW and YD).

\section{Author contributions}

LL and ZW designed the research; ZW, LQ, SW and YD provided financial support; LQ, SW, YD, KH, YL, LW, LL, IL, LG provide study material; LL and IL collected and/or assembled of data; LL and IL analysised data; LL, LQ and ZW wrote the manuscript; ZW final approved of manuscript.

\section{References}

1 Mozaffarian D, Benjamin EJ, Go AS et al. Heart disease and stroke statistics--2015 update: a report from the American Heart Association. Circulation 2015; 131: e29-322.

2 Laflamme MA, Murry CE. Regenerating the heart. Nat Biotechnol 2005; 23: 845-856.

3 Pouliopoulos J, Chik WW, Kanthan A et al. Intramyocardial adiposity after myocardial infarction: new 
implications of a substrate for ventricular tachycardia. Circulation 2013; 128: 2296-2308.

4 Zhang H, Pu W, Liu Q et al. Endocardium contributes to cardiac fat. Circ Res 2016; 118: 254-265.

5 Ieda $\mathrm{M}, \mathrm{Fu}$ JD, Delgado-Olguin $\mathrm{P}$ et al. Direct reprogramming of fibroblasts into functional cardiomyocytes by defined factors. Cell 2010; 142: 375-386.

6 Qian L, Huang Y, Spencer CI et al. In vivo reprogramming of murine cardiac fibroblasts into induced cardiomyocytes. Nature 2012; 485: 593-598.

7 Wang L, Liu Z, Yin C et al. Stoichiometry of Gata4, Mef2c, and Tbx5 influences the efficiency and quality of induced cardiac myocyte reprogramming. Circ Res 2015; 116: $237-244$.

8 Nam YJ, Song K, Luo X et al. Reprogramming of human fibroblasts toward a cardiac fate. Proc Natl Acad Sci USA 2013; 110: 5588-5593.

9 Song K, Nam YJ, Luo X et al. Heart repair by reprogramming non-myocytes with cardiac transcription factors. Nature 2012; 485: 599-604.

10 Addis RC, Ifkovits JL, Pinto F et al. Optimization of direct fibroblast reprogramming to cardiomyocytes using calcium activity as a functional measure of success. $\mathrm{J} \mathrm{Mol} \mathrm{Cell}$ Cardiol 2013; 60: 97-106.

11 Christoforou N, Chellappan M, Adler AF et al. Transcription factors MYOCD, SRF, Mesp1 and SMARCD3 enhance the cardio-inducing effect of GATA4, TBX5, and MEF2C during direct cellular reprogramming. PloS ONE 2013; 8: e63577.

12 Zhou H, Dickson ME, Kim MS, Bassel-Duby R, Olson EN. Akt1/protein kinase B enhances transcriptional reprogramming of fibroblasts to functional cardiomyocytes. Proc Natl Acad Sci USA 2015; 112: 11864-11869.

13 Zhao $\mathrm{Y}$, Londono $\mathrm{P}$, Cao $\mathrm{Y}$ et al. High-efficiency reprogramming of fibroblasts into cardiomyocytes requires suppression of pro-fibrotic signalling. Nat Commun 2015; 6: 8243.

14 Muraoka N, Yamakawa H, Miyamoto K et al. MiR-133 promotes cardiac reprogramming by directly repressing Snail and silencing fibroblast signatures. EMBO J 2014; 33: $1565-1581$.

15 Jayawardena TM, Egemnazarov B, Finch EA et al. MicroRNA-mediated in vitro and in vivo direct reprogramming of cardiac fibroblasts to cardiomyocytes. Circ Res 2012; 110: 1465-1473.

16 Liu Z, Chen O, Zheng $\mathrm{M}$ et al. Re-patterning of H3K27me3, H3K4me3 and DNA methylation during fibroblast conversion into induced cardiomyocytes. Stem Cell Res 2016; 16: 507-518.

17 Hirai H, Kikyo N. Inhibitors of suppressive histone modification promote direct reprogramming of fibroblasts to cardiomyocyte-like cells. Cardiovasc Res 2014; 102: 188-190.

18 Zhou Y, Wang L, Vaseghi HR et al. Bmil is a key epigenetic barrier to direct cardiac reprogramming. Cell Stem Cell 2016; 18: 382-395.

19 Treutlein B, Lee QY, Camp JG et al. Dissecting direct reprogramming from fibroblast to neuron using single-cell RNA-seq. Nature 2016; 534: 391-395.
20 Parenti A, Halbisen MA, Wang K, Latham K, Ralston A. OSKM induce extraembryonic endoderm stem cells in parallel to induced pluripotent stem cells. Stem Cell Rep 2016; 6: 447-455.

21 Zhou L, Liu Y, Lu L, Lu X, Dixon RA. Cardiac gene activation analysis in mammalian non-myoblasic cells by Nkx2-5, Tbx5, Gata4 and Myocd. PloS ONE 2012; 7: e48028.

22 Luna-Zurita L, Stirnimann CU, Glatt S et al. Complex Interdependence Regulates Heterotypic Transcription Factor Distribution and Coordinates Cardiogenesis. Cell 2016; 164: 999-1014.

23 Wamstad JA, Alexander JM, Truty RM et al. Dynamic and coordinated epigenetic regulation of developmental transitions in the cardiac lineage. Cell 2012; 151: 206-220.

24 Yang YJ, Song TY, Park J et al. Menin mediates epigenetic regulation via histone $\mathrm{H} 3$ lysine 9 methylation. Cell Death Dis 2013; 4: e583.

25 Agarwal SK, Jothi R. Genome-wide characterization of menin-dependent $\mathrm{H} 3 \mathrm{~K} 4 \mathrm{me} 3$ reveals a specific role for menin in the regulation of genes implicated in MEN1-like tumors. PloS ONE 2012; 7: e37952.

26 Sidler C, Woycicki R, Li D, Wang B, Kovalchuk I, Kovalchuk O. A role for SUV39H1-mediated H3K9 trimethylation in the control of genome stability and senescence in WI38 human diploid lung fibroblasts. Aging 2014; 6: 545-563.

27 Greiner D, Bonaldi T, Eskeland R, Roemer E, Imhof A. Identification of a specific inhibitor of the histone methyltransferase SU(VAR)3-9. Nat Chem Biol 2005; 1: 143-145.

28 Borkin D, He S, Miao H et al. Pharmacologic inhibition of the Menin-MLL interaction blocks progression of MLL leukemia in vivo. Cancer Cell 2015; 27: 589-602.

29 Hacer Karatas, Jiao Ji, Shirley Lee et al. Structure-based design, synthesis and evaluation of cyclic peptidomimetics blocking the MLL-WDR5 protein-protein interaction. $J$ Med Chem 2016 (in press).

30 Cao F, Townsend EC, Karatas $\mathrm{H}$ et al. Targeting MLL1 $\mathrm{H} 3 \mathrm{~K} 4$ methyltransferase activity in mixed-lineage leukemia. Mol Cell 2014; 53: 247-261.

31 Wang L, Liu Z, Yin C, Zhou Y, Liu J, Qian L. Improved generation of induced cardiomyocytes using a polycistronic construct expressing optimal ratio of Gata4, Mef2c and Tbx5. J Vis Exp. (e-pub ahead of print 13 November 2015; doi:10.3791/53426).

32 Wang P, Lin C, Smith ER et al. Global analysis of H3K4 methylation defines MLL family member targets and points to a role for MLL1-mediated H3K4 methylation in the regulation of transcriptional initiation by RNA polymerase II. Mol Cell Biol 2009; 29: 6074-6085.

33 Zhang $\mathrm{H}$, Gayen S, Xiong $\mathrm{J}$ et al. MLL1 inhibition reprograms epiblast stem cells to naive pluripotency. Cell Stem Cell 2016; 18: 481-494.

34 Mahmoudi T, Verrijzer CP. Chromatin silencing and activation by Polycomb and trithorax group proteins. Oncogene 2001; 20: 3055-3066. 
35 Jimenez MA, Akerblad P, Sigvardsson M, Rosen ED. Critical role for Ebf1 and Ebf2 in the adipogenic transcriptional cascade. Mol Cell Biol 2007; 27: 743-757.

36 Dreijerink KM, Varier RA, van Beekum $\mathrm{O}$ et al. The multiple endocrine neoplasia type 1 (MEN1) tumor suppressor regulates peroxisome proliferator-activated receptor gamma-dependent adipocyte differentiation. Mol Cell Biol 2009; 29: 5060-5069.

37 Jumabay M, Zhang R, Yao Y, Goldhaber JI, Bostrom KI. Spontaneously beating cardiomyocytes derived from white mature adipocytes. Cardiovasc Res 2010; 85: 17-27.

38 Choi YS, Dusting GJ, Stubbs $\mathrm{S}$ et al. Differentiation of human adipose-derived stem cells into beating cardiomyocytes. J Cell Mol Med 2010; 14: 878-889.

$39 \mathrm{Fu} \mathrm{Y}$, Huang $\mathrm{C}, \mathrm{Xu} \mathrm{X}$ et al. Direct reprogramming of mouse fibroblasts into cardiomyocytes with chemical cocktails. Cell Res 2015; 25: 1013-1024.
40 Cao N, Huang Y, Zheng J et al. Conversion of human fibroblasts into functional cardiomyocytes by small molecules. Science 2016; 352: 1216-1220.

(Supplementary information is linked to the online version of the paper on the Cell Discovery website.)

(c) (i) This work is licensed under a Creative Commons Attribution 4.0 International License. The images or other third party material in this article are included in the article's Creative Commons license, unless indicated otherwise in the credit line; if the material is not included under the Creative Commons license, users will need to obtain permission from the license holder to reproduce the material. To view a copy of this license, visit http://creativecommons.org/licenses/by/4.0/

(C) The Author(s) 2016 